Results Women ever exposed to benzene had a significantly elevated risk of NHL (Hazard Ratio $(\mathrm{HR})=1.87,95 \% \mathrm{CI}=$ 1.19-2.96). Compared to unexposed women, significant trends in NHL risk were observed for increasing years of benzene exposure $\left(\mathrm{p}_{\text {trend }}=0.009\right.$ ) and increasing cumulative exposure levels $\left(\mathrm{p}_{\text {trend }}=0.01\right)$, with women in the highest duration and cumulative exposure tertiles having a significantly elevated association with NHL $(\mathrm{HR}=2.07,95 \% \mathrm{CI}=1.07-4.01$ and $\mathrm{HR}=$ $2.16,95 \%$ CI $=1.17-3.98$, respectively).

Conclusions Our study is the first to our knowledge to evaluate this association in the context of a population-based prospective cohort of all women with diverse occupational histories. Our findings add to the evidence that benzene is associated with risk of NHL.

\section{A STUDY OF RADIOGRAPHIC ABNORMALITIES IN MINNESOTA TACONITE WORKERS}

Jeff Mandel, Christine Lambert, Bruce Alexander, Richard MacLehose, Gurumurthy Ramachandran. University of Minnesota, Minneapolis, USA

\subsection{6/oemed-2014-102362.126}

Objectives A limited number of studies have been conducted in the taconite industry, typically without detailed exe posure information. We assessed occupational exposure to elongate mineral particles (EMP) in association with chest $x$-ray findings in a cross-sectional screening of 1188 current and former taconite workers.

Method Exposure was determined by a combination of some 1300 onsite measures taken by researchers and historical measures taken by mining companies. The NIOSH 7400 measurement method (PCM) was used to count EMP. Twenty-eight similarly exposed groupings (SEG) were developed and contained all jobs. Each SEG had multiple EMP measures as an anchoring point for historical exposure estimates. Work histories were standardised, mapped to SEGs and used to estimate exposures for each worker in each SEG. Chest x-rays were obtained using ILO methods, with two independent radiologists. Prevalence odds ratios were estimated by logistic regression for work duration and cumulative EMP exposure, adjusting for age, gender, BMI and occupations with high potential for asbestos.

Results Pleural findings occurred in $16.8 \%$ by consensus. Abnormalities were associated with duration of employment in the taconite industry for those working 21 years or more $(\mathrm{OR}=1.59,95 \% \mathrm{CI}=1.06-2.40)$. Pleural abnormalities were also associted with cumulative exposure to EMP for those cumulatively exposed to $1.16 \mathrm{EMP} / \mathrm{cc}$-years or more $(\mathrm{OR}=1.93,95 \%$ $\mathrm{CI}=1.32-2.83)$. Associations between parenchymal abnormalities and exposure were not observed.

Conclusions This study suggests that pleural abnormalities are related to duration of employment and to EMP exposure in taconite miners. Due to the measurement method, the specific type of EMP could not be described further.

\section{THE CONTRIBUTION OF OCCUPATIONAL STUDIES TO THE IDENTIFICATION OF CARCINOGENS IN THE IARC MONOGRAPHS AND HOW TO FURTHER IMPROVE IT}

Dana Loomis, Neela Guha, Kurt Straif. IARC, Lyon, France

10.1136/oemed-2014-102362.127

Objectives To discuss the important role of occupational studies in identifying carcinogens and suggest how it could be still greater.
Method The contribution of occupational studies to the IARC monographs is reviewed.

Results Occupational epidemiology has made important contributions beyond developing knowledge to protect workers' health, notably in identifying carcinogens of concern for the general population. The IARC Monographs have evaluated many carcinogens for which occupational studies have provided key evidence. The recent classifications of diesel engine exhaust, trichloroethylene and polychlorinated biphenyls (PCBs) as human carcinogens, which depended heavily on data from occupational studies, are illustrative. In the evaluation of PCBs, for example, occupational cohort studies showing an exposure-related increase in the risk of malignant melanoma were pivotal for the conclusion of sufficient evidence of carcinogenicity. Despite such noteworthy contributions, the number of occupational studies that are ultimately informative tends to be relatively small relative to the number reviewed. The most informative studies tend to have common features, including clear reporting of methods and results, well-defined outcomes, quantitative estimates of exposure, adequate control of major confounders, and state of the art analytical methods, often with internal analyses of exposure-response. In contrast, studies that are too broadly focused and those with crude classifications of exposure or outcome, analyses by external comparisons alone or poor reporting of the methods and results are often less informative in the final evaluation.

Conclusions While occupational studies are important for carcinogen identification, their relevance could be further enhanced with improvements in study design, methods and reporting.

\section{YEARS (1989-2008) OF DIRECT COSTS ASSOCIATED WITH FALLS FROM HEIGHT AMONG UNION CARPENTERS, WASHINGTON STATE, USA}

${ }^{1}$ Hester Lipscomb, ${ }^{1}$ Ashley Schoenfisch, ${ }^{2}$ Wilfrid Cameron, ${ }^{3}$ Kristen Kucera, ${ }^{4}$ Darrin Adams, ${ }^{4}$ Barbara Silverstein. ${ }^{1}$ Duke University, Durham, NC, USA; ${ }^{2}$ Strategic Solutions for Safety, Health and Environment, Seattle, Washington, USA; ${ }^{3}$ University of NC, Chapel Hill, NC, USA; ${ }^{4}$ SHARP, Department of Labor and Industries, Olympia, Washington, USA

\subsection{6/oemed-2014-102362.128}

Objectives To examine trends in workers' compensation payments for falls from height (FFH) among a large cohort of carpenters over a 20-year period (1989-2008). Cost data provide an important metric reflecting frequency of falls and severity of associated injuries.

Method Using combined administrative data we evaluated workers' compensation (WC) payments associated with FFH among a large ( $\mathrm{n}=24$ 830) 20-year cohort (1989-2008) of union carpenters in Washington State. Mean payments, costs rates and adjusted rate ratios based on hours worked were calculated using negative binomial regression to evaluate cost patterns based on age, union tenure, type of carpentry work and calendar time after adjusting and discounting to 2011 dollar values.

Results FFH accounted for $\$ 66.6$ million in WC payments (a burden of $\$ 0.35$ per hour worked) over the 20-year period. FFH were responsible for $5.5 \%$ of injuries but $15.1 \%$ of costs. Marked cost declines were observed over time, but not in a monotonic fashion. Reductions were more pronounced for indemnity than for medical care. Mean costs per fall were unchanged in the latter years of observation from those observed 1995-1996. Individuals performing millwrighting or drywall installation had cost rates over twice as high as commercial construction. Mean costs were 2.3 times greater among carpenters 
over 50 than those under 30 , but their cost rates were only modestly higher.

Conclusions Progress has been made in reducing direct costs associated with FFH in this cohort particularly though 1996, but primary gains reflect the reduction in frequency while FFH that occur remain serious.

\section{PESTICIDE EXPOSURE DURING RE-ENTRY TASKS AND HARVESTING IN VINEYARDS: RESULTS OF THE PESTEXPO PROGRAM}

1,2I Isabelle Baldi, ${ }^{3,4}$ Pierre Lebailly, ${ }^{1}$ Ghislaine Bouvier, ${ }^{1}$ Virginie Rondeau, ${ }^{5}$ Valérie Bouchart, ${ }^{1}$ Mireille Canal-Raffin, ${ }^{1}$ Alain Garrigou. ${ }^{1}$ Univ. Bordeaux, ISPED, Laboratoire Santé Travail Environnement, Centre INSERM U 897 Epidemiologie-Biostatistique, F-33000 Bordeaux, France; ${ }^{2}$ CHU de Bordeaux, Service de Médecine Du Travail, F-33000 Bordeaux, France; ${ }^{3}$ INSERM, UMR 1086-Cancers Et Préventions, F-14000 Caen, France; ${ }^{4}$ Centre François Baclesse, F-14000 Caen, France; ${ }^{5}$ Laboratoire Départemental Frank Duncombe, F-14000 Caen, France

\subsection{6/oemed-2014-102362.129}

Objectives Physical contact with treated crops, animals or other surfaces is responsible for the transfer of pesticides to the worker's skin in agricultural tasks and makes their cutaneous absorption possible. In the Bordeaux area (France), the PESTEXPO study described levels of pesticide exposure and identified their determinants during re-entry and harvesting in vineyards.

Method Between 2002 and 2007, 46 days of work involving reentry tasks and 48 harvesting days were observed to analyse exposures to dithiocarbamates or folpet. The potential determinants were generated from the following parameters collected on standardised forms during field observations: i)general conditions of the task, ii)operator characteristics, iii)estate characteristics, iv) task conditions and v)characteristics of the last treatment involving folpet or dithiocarbamates, including delay since treatment. Dermal contamination was assessed using patches placed on the skin and hand-washing at the end of each working phase. Results Daily median contamination was $1967.7 \mu \mathrm{l}$ of mixture during re-entry (90e percentile: $5045.3 \mu \mathrm{l}$ ) and $18.7 \mu \mathrm{l}$ during harvesting $(90 \mathrm{e}$ percentile: $911.4 \mu \mathrm{l})$. Contamination level was strongly correlated to the type of task. For re-entry, the highest contaminations were observed during raising of wires and cutting of branches. During the harvest, the contamination was maximal for grape-picking. The delay since the last treatment and the rate of active ingredient per hectare played a role, together with meteorological factors, crop and farm characteristics, gloves and clothes.

Conclusions Our results underline the necessity to take into account exposures during re-entry and harvest when considering pesticide exposure, both for epidemiological research and preventive action.

\section{OCCUPATIONAL EXPOSURE TO LEAD AND CANCER IN TWO COHORT STUDIES OF MEN AND WOMEN IN SHANGHAI, CHINA}

${ }^{1}$ Linda M Liao, ${ }^{1}$ Melissa C Friesen, ${ }^{2}$ Yong-Bing Xiang, ${ }^{3}$ Hui Cai, ${ }^{4}$ Dong-Hee Koh, ${ }^{1}$ Bu-Tian Ji, ${ }^{3}$ Gong Yang, ${ }^{2}$ Hong-Lan Li, ${ }^{1}$ Sarah J Locke, ${ }^{1}$ Nathaniel Rothman, ${ }^{3}$ Wei Zheng, ${ }^{2}$ Yu-Tang Gao, ${ }^{3}$ Xiao-Ou Shu, ${ }^{1}$ Mark P Purdue. ${ }^{1}$ Division of Cancer Epidemiology and Genetics, National Cancer Institute, Bethesda, MD, USA; ${ }^{2}$ Department of Epidemiology, Shanghai Cancer Institute, Shanghai, China; ${ }^{3}$ Department of Medicine, Vanderbilt University, School of Medicine, Nashville, TN, USA; ${ }^{4}$ Carcinogenic Hazard Branch, National Cancer Center, Seoul, Republic of Korea

10.1136/oemed-2014-102362.130
Objectives Epidemiologic studies of occupational lead exposure have suggested increased risks of cancers of the brain, kidney, lung, meninges, and stomach; however, the totality of the evidence is inconsistent. To clarify whether lead is a carcinogen, we investigated the relationship between occupational lead exposure and risks of these five cancer sites in two prospective cohort studies in Shanghai, China.

Method Annual job/industry-specific estimates of lead fume and lead dust exposure were derived from a statistical model that combined expert ratings of lead intensity with inspection measurements collected by the Shanghai Centre for Disease Control and Prevention. The job/industry estimates were applied to the lifetime work histories of subjects from the Shanghai Women's Health Study (73 363 participants) and the Shanghai Men's Health Study (61 379 participants) to estimate cumulative exposure to lead dust and lead fume. Cohort-specific relative hazard rate ratios (RRs) and 95\% confidence intervals (CI) were estimated using Cox proportional hazards regression models and then pooled using a random effects meta-analysis model.

Results We observed a statistically significant increased risk of meningioma among individuals with estimated occupational exposure to lead dust or fumes ( $\mathrm{RR}=2.4,95 \% \mathrm{CI}: 1.1-5.0)$, and in particular among those with an above-median cumulative exposure to dust or fumes ( $R R=3.1,95 \% \mathrm{CI}: 1.3-7.4)$. We observed suggestive associations with lead exposure for cancers of the kidney ( $\mathrm{RR}=1.4,95 \% \mathrm{CI}: 0.9-2.3)$ and brain $(\mathrm{RR}=1.8$, 95\% CI:0.7-4.8), and null findings for cancers of the lung and stomach.

Conclusions Our findings provide additional evidence that occupational lead exposure increases risk of meningioma.

\section{POSSIBLE PRO-CARCINOGENIC EFFECT OF ENDOTOXIN ON LUNG CANCER IN AN EXTENDED FOLLOW-UP OF SHANGHAI WOMEN TEXTILE WORKERS}

${ }^{1}$ Harvey Checkoway, ${ }^{2}$ Jessica Lundin, ${ }^{3}$ Sadie Costello, ${ }^{4}$ Roberta Ray, ${ }^{4}$ Wenjin Li, ${ }^{3}$ Ellen Eisen, ${ }^{5}$ George Astrakianakis, ${ }^{2}$ Noah Seixas, ${ }^{6}$ Kate Applebaum, ${ }^{7}$ Dao Li Gao, ${ }^{4}$ David Thomas. ${ }^{1}$ University of California, San Diego, La Jolla, CA, USA; ${ }^{2}$ University of Washington, Seattle, WA, USA; ${ }^{3}$ University of California, Berkeley, Berkeley, CA, USA; ${ }^{4}$ Fred Hutchinson Cancer Research Center, Seattle, WA, USA; ${ }^{5}$ University of British Columbia, Vancouver, BC, Canada; ${ }^{6}$ George Washington University, Washington, DC, USA; 'Zhong Shan Hospital, Shanghai, China

\subsection{6/oemed-2014-102362.131}

Objectives To examine further the association between endotoxin and risk of lung cancer among Shanghai women textile workers in an extended follow-up of the cohort. The initial follow-up indicated an inverse exposure-response relation.

Method We updated a case-cohort study nested within a cohort of 267400 women. We compared exposure histories of 1456 incident lung cancers cases diagnosed during 1989-2006 with those of a reference subcohort of 3022 workers who were free of lung cancer at the end of follow-up. Endotoxin exposures were based on a quantitative job/exposure matrix. Relative risks (hazard ratios [HR]) associated with cumulative exposure, adjusted for age and smoking history, were estimated by Cox proportional hazards modelling adapted for the case-cohort design. We conducted exposure-response trend analyses for cumulative exposures lagged by 0,10 , and 20 years, and separately for time windows of $<15$ and $>15$ years since first exposure.

Results Overall, we observed no associations between cumulative exposure and lung cancer. In contrast, analyses by exposure 